Accepted for publication: The Astrophysical Journal

\title{
Direct Evidence for an Enhancement of Helium in Giant Stars in Omega Centauri ${ }^{1}$
}

\author{
A. K. Dupree \\ Harvard-Smithsonian Center for Astrophysics, 60 Garden Street, Cambridge, MA 02138 \\ dupree@cfa.harvard.edu \\ Jay Strader ${ }^{2}$ \\ Harvard-Smithsonian Center for Astrophysics, 60 Garden Street, Cambridge, MA 02138 \\ jstrader@cfa.harvard.edu \\ Graeme H. Smith \\ University of California Observatories/Lick Observatory, University of California, Santa \\ Cruz, CA 95064 \\ graeme@ucolick.org
}

\begin{abstract}
The double main sequence identified in the globular cluster Omega Centauri has been interpreted using isochrones to indicate a large variation in the abundance of helium. If true, a helium enhancement carries strong implications for the chemical and stellar evolutionary history of this cluster. However, only indirect measures currently support this conjecture. We report the discovery of a variation in the line strength of the near-infrared He I $10830 \AA$ transition in twelve similar red giants in Omega Centauri observed with PHOENIX on Gemini-S. Abundances of these stars derived from Magellan/MIKE spectra taken at Las Campanas Observatory show that the helium transition is not detected in the most metal-poor population $([\mathrm{Fe} / \mathrm{H}]<-1.8)$, yet is present in the majority of stars with $[\mathrm{Fe} / \mathrm{H}] \geq-1.8$. These observations give the first direct evidence for an enhancement of helium in Omega Centauri. The appearance of helium appears better correlated with increased $[\mathrm{Al} / \mathrm{Fe}]$ and $[\mathrm{Na} / \mathrm{Fe}]$ abundances than as a function of $[\mathrm{Fe} / \mathrm{H}]$, giving observational support to the presence of high-temperature H-burning in a prior generation of stars.
\end{abstract}


Subject headings: globular clusters: general - globular clusters: individual (Omega Centauri) — stars: Population II — stars: abundances

\section{Introduction}

Omega Centauri ( $\omega$ Cen) is a globular cluster long known to have a color spread along the red giant branch (Woolley 1966; Geyer 1967) indicative of a spread in the $[\mathrm{Fe} / \mathrm{H}]$ abundances as originally identified in cluster RR Lyrae variables (Freeman \& Rodgers 1975). Among subgiants too, four (and possibly five) subgroups of different ages and metallicities have been identified (Villanova et al. 2007; Bellini et al. 2010). The discovery (Anderson 1997; Bedin et al. 2004) of multiple stellar populations on the main sequence in a globular cluster, first identified in $\omega$ Cen was possibly a singular anomaly. But another globular cluster, NGC 2808, displays multiple branches on the main sequence (Piotto et al. 2007). These results, based on unevolved dwarf stars, present firm evidence for multiple episodes of star formation in a globular cluster, and upset the long-held paradigm that a globular cluster consists of a coeval population of stars (Piotto 2009).

Norris (2004) offered an explanation for the 'double' main sequence in Omega Cen based on stellar models, as resulting from a substantially enhanced helium abundance in the 'blue' main sequence $(\Delta Y=+0.13 ; Y=0.38)$, over the primordial abundance $(Y=0.25)$ in the 'red' sequence. Subsequently, the blue main-sequence of stars in $\omega$ Cen was discovered to be more metal-rich than the red main sequence $\left([\mathrm{M} / \mathrm{H}]_{b l u e}=-1.26\right.$ vs $[\mathrm{M} / \mathrm{H}]_{\text {red }}=-1.57$; Piotto et al. 2005). The cluster NGC 2808 shows a similar effect (Bragaglia et al. 2010a).

An enhanced helium abundance would have a number of ramifications. Stellar models suggest that excess helium could account for many characteristics of globular cluster color-magnitude diagrams: an extended horizontal branch (HB), gaps in the HB, RR Lyr periods and period distributions, and the ratio of blue to red HB stars (D'Antona \& Caloi 2004, 2008; Norris 2004; Catelan 2009; Gratton et al. 2010). It is currently thought that a

\footnotetext{
${ }^{1}$ Data presented herein were obtained at the Gemini Observatory, which is operated by the Association of Universities for Research in Astronomy, Inc., under a cooperative agreement with the NSF on behalf of the Gemini partnership: the National Science Foundation (United States), the Science and Technology Facilities Council (United Kingdom), the National Research Council (Canada), CONICYT (Chile), the Australian Research Council (Australia), Ministério da Ciência e Tecnologia (Brazil) and Ministerio de Ciencia, Tecnología e Innovación Productiva (Argentina). This paper also includes spectra gathered with the 6.5-meter Magellan Telescope/CLAY located at Las Campanas Observatory, Chile.

${ }^{2}$ Hubble Fellow, now Menzel Fellow at Harvard College Observatory, Cambridge, MA USA.
} 
helium enhancement and other chemical inhomogeneities might result from self-enrichment (pollution) of a second generation of cluster stars by the processed material from the first generation stars. Various sources for excess helium have been proposed: production by first generation asymptotic branch stars (AGB) of intermediate mass, or massive hot stars; and Type II supernovae. However models can not uniquely identify the source of the enrichment; concerns remain because all of the processed first generation material is required to produce enrichment, and the material from large numbers of stars must be homogenized (Norris 2004; Piotto et al. 2005). Omega Cen appears to have an anomalously blue HB (D'Cruz et al. 2000; Momany et al. 2004) which has been attributed (Norris 2004; Piotto et al. 2005) to the progeny of the blue main sequence. However, AGB models (Choi \& Yi 2008) can not produce the quantity of helium in first generation stars as required for Omega Cen.

While there is great interest in the possibility of a helium enhancement in these multiple populations, only indirect methods have been used to date to infer this enhancement in globular cluster stars. The observable quantities rely principally on the appearance of the color magnitude diagrams, comparison with stellar isochrones, and the composition of elements other than helium, in particular $\mathrm{Na}$ and $\mathrm{Al}$, which signal the products of high-temperature H-burning (Densikov \& Denisenkova 1989; Langer et al. 1993) including CNO, NeNa, and $\mathrm{MgAl}$ cycles that lead to helium production (cf. Gratton et al. 2001; Gratton et al. 2004; Bragaglia et al. 2010b).

Helium is a difficult element to detect spectroscopically in cool stars because high temperatures are generally required to produce the optical transitions. Hot stars, such as the HB objects, where helium might appear, are subject to diffusion and preferential settling of elements making the determination of abundances ambiguous (Da Costa et al. 1986; Behr et al. 1999). However, the infrared He I transition at $10830 \AA$, has been detected in metal-poor red giant stars in the field (Dupree et al. 1992, 2009) as well as in the globular cluster M13 (Smith et al. 2004). The strength of the helium absorption is independent of $[\mathrm{Fe} / \mathrm{H}]$ metallicity in the field-star sample (Dupree et al. 2009). A calculation of the $10830 \AA$ profile in the quiet sun with PANDORA (Avrett \& Loeser 2008) demonstrates that a change in the helium abundance from $\mathrm{Y}=0.28$ to $\mathrm{Y}=0.4$ increases the equivalent width of the absorption by a factor of 3 . Thus a simple measure of the distribution of equivalent widths in stars within a restricted region of temperature and luminosity could reveal a variation in helium strength. We report the results of such a study here of stars in the globular cluster $\omega$ Cen. Because this cluster is believed to have substantial helium enhancement (Norris 2004), it is an optimum target.

Spectroscopic observations of $\omega$ Cen giants at Gemini-S (for the near-IR He I transition) and Magellan/Clay (for optical spectra) are presented in Section 2; the derivation of 
abundances from Magellan spectra is addressed in Section 3; results are discussed in Section 4, and final comments in Section 5.

\section{Observations and Reduction}

Selection of target stars in $\omega$ Cen was guided by the fact that the He I $\lambda 10830$ transition appears in metal-poor field giants with $T_{\text {eff }} \geq 4500 \mathrm{~K}$ where the stellar chromospheres reach temperatures $(\mathrm{T} \sim 10,000 \mathrm{~K}$ ) high enough to produce the helium line (Dupree et al. 2009). A restricted region of the red giant branch was selected to fit the temperature criterion. The target region spanned 0.05 in $B-V$ color and was limited to a range of $\sim 0.5$ magnitudes in $\mathrm{V}$ between 12.95 and 13.48 (Fig. 1). This selection avoids asymptotic giant branch stars and minimizes or eliminates any temperature or luminosity dependencies of the line strength. In addition, horizontal branch stars are avoided to eliminate objects where elemental diffusion might occur. All of the target stars have a membership probability of 98 to $100 \%$ based on proper motion studies (van Leeuwen et al. 2000), and our radial velocity measures, discussed later, are consistent with membership as well.

\subsection{Gemini-S/PHOENIX Infrared Spectra}

Classical time was awarded (Program GS-2010A-C-7) for 3 nights on PHOENIX 3 mounted on Gemini-S (Hinkle et al. 2003) to obtain high-resolution spectra of the He I $10830 \AA$ line. Three clear nights of observations were obtained beginning 24 March 2010 UT. The PHOENIX setup consisted of a slit width of 4 pixels yielding a spectral resolution of $\sim 50,000$. The order-sorting filter J9232 was selected which spans $1.077-1.089 \mu \mathrm{m}$, and allows access to the He I $1.083 \mu \mathrm{m}$ line which is shifted by $\sim+230 \mathrm{~km} \mathrm{~s}^{-1}$ (equivalent to $8.3 \AA$ ) due to the radial velocity of the $\omega$ Cen cluster. Standard procedures were followed by acquiring target spectra using a nodding mode (A-B) with a spatial separation of 5 arcsec. Standard stars and telluric comparison objects were observed with a nodding pattern using a separation of 8 arcsec to avoid contamination of the targets by a residual image. Exposure times for most of the $\omega$ Cen giants totaled 2 hours, and 1 hour for two of the brightest targets. To verify the grating setup, a spectrum of a nearby star with strong P Cygni-type emission at $10830 \AA$ was obtained. Because standard comparison lamps have a sparse wavelength pattern in this near-infrared region, we observed a bright Population I K giant containing many securely identified narrow absorption lines in order to determine the wavelength scale. Throughout

\footnotetext{
${ }^{3}$ See http://www.noao.edu/ngsc/phoenix/phoenix.html
} 
the night, spectra of fast-rotating B stars were obtained at various air masses to monitor the strength of the telluric water vapor features. Flat exposures and dark exposures were taken at the end of each night. The twelve red giants comprising the targets in $\omega$ Cen are listed in Table 1.

A technical issue with PHOENIX became apparent during our run at Gemini-S. During the periods of good seeing, $(\sim 0.4$ arcsec $)$, the spectrum appeared double in the spatial direction, leading to slightly degraded image quality. The source of this doubling is not now known 4 Other than a moderate reduction in the $\mathrm{S} / \mathrm{N}$, this issue does not affect any of our results.

The raw images were corrected for cosmic rays using L.A.Cosmic (van Dokkum 2001). After this, the nodded image pairs were subtracted from each other. These subtracted images were divided by a dark-corrected, normalized flat field, followed by optimal extraction of onedimensional spectra. Owing to the paucity of arc lines in the region of the $10830 \AA$ line, the spectra were wavelength calibrated using observations of strong-lined K giants with known radial velocities. The individual calibrated spectra were combined with proper variance weighting. The spectra were normalized by excluding the region between $10833-10844 \AA$ where the Doppler-shifted Si I line $(\lambda 10827.09)$ and He I line $(\lambda 10830.34)$ occur, and fitting a second-order cubic spline to the spectra. Spectra of the helium line region in the target giants are shown in Fig. 2.

Equivalent widths of the He I absorption were measured from the unbinned data. Spectra were not corrected for telluric absorption, but the equivalent width of a water vapor line, if blended with helium, was subtracted from the total helium absorption. Our spectra of rapidly rotating $\mathrm{B}$ stars, taken as standard stars, display a correlation between the equivalent width of the strongest water vapor line in this region, $\lambda_{\text {air }}=10832.109 \AA$ (Breckinridge \& Hall 1973) and the $10838.034 \AA$ line which in some stars blends with the helium transition. A straight line represents the correlation with a $\pm 2 \mathrm{~m} \AA(1 \sigma)$ error of the fit. This correlation was used to estimate the strength of $10838.034 \AA$ in the target stars based on the observed

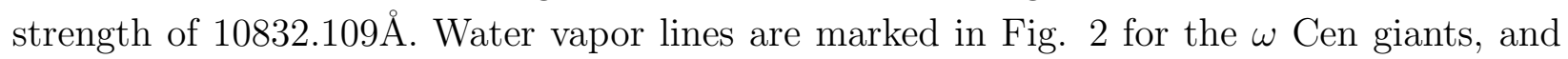
a sample of the telluric standard stars is shown in Fig. 3. The water vapor transition at $10838.034 \AA$ varied in strength from 4 to $18 \mathrm{~m} \AA$. Where helium is not apparent in our targets,

\footnotetext{
${ }^{4}$ The doubling phenomenon has been observed at Gemini-S since PHOENIX began operation on Gemini-S in 2002 (B. Rodgers, private communication). Two possibilities have been suggested to us: the telescope was slightly out of focus on the slit (K. Hinkle, private communication) or an astigmatic collimator whose effects become substantially more pronounced at longer wavelengths as shown in PHOENIX spectra obtained at the KPNO 2.1 coudé feed in 1998 (C. Kulesa, private communication). We assume this was not noticed previously since, in queue mode, PHOENIX was not likely to be used during optimum seeing conditions.
} 
the equivalent width in Table 1 represents an upper limit to the strength of a non-observed helium line, and generally corresponds to the equivalent width of the water vapor transition.

Radial velocities of the targets were determined from the high resolution infrared spectra, based on fitting the Si I photospheric absorption line with a gaussian profile. We also measured central wavelengths of 10-13 Fe II lines for each star from the MIKE spectra for the abundance analysis discussed later, and velocity measures from the individual lines have a dispersion of less than $1.7 \mathrm{~km} \mathrm{~s}^{-1}$. The Fe II velocities agree with the results from Si I to better than $0.2 \mathrm{~km} \mathrm{~s}^{-1}$. Values of the radial velocity are listed in Table 1 . The mean radial velocity of $\omega$ Cen has a distribution peaked around $+233 \mathrm{~km} \mathrm{~s}^{-1}$ (Reijns et al. 2006). Our targets are clearly radial velocity members of the cluster confirming the membership determination from proper motions (van Leeuwen et al. 2000). For the nine targets in common with Reijns et al. (2006), our values agree to better than $2 \mathrm{~km} \mathrm{~s}^{-1}$, with the exception of LEID 54064 where we differ by $8.1 \mathrm{~km} \mathrm{~s}^{-1}$; this star has the largest error of $4.7 \mathrm{~km} \mathrm{~s}^{-1}$ reported by Reijns et al. (2006).

\subsection{Magellan/MIKE spectra}

Echelle spectra of the PHOENIX targets were obtained at the Magellan/Clay telescope on 30 June - 2 July 2010 (UT) using the MIKE spectrograph (Bernstein et al. 2002) with a $0.70 \times 5$ arcsec slit. The spectral resolution with this setup ranges from $\sim 26,000$ on the blue side $(\lambda \lambda 3350-5000)$ to $\sim 36,000$ on the red side $(\lambda \lambda 4900-9300)$. Total exposure times on individual targets varied from 40 minutes to 1 hour, generally divided between two exposures. An IDL pipeline developed by S. Burles, R. Bernstein, and J. S. Prochaska5 was used to extract the spectra. This efficient pipeline evaluates the detector gain, processes the milky and order-tracing flats, obtains the Th-Ar arc solution, constructs a slit flat, and extracts a spectrum including sky subtraction. IRAF procedures were used to flatten individual echelle orders, and combine multiple exposures. The signal-to-noise ratio ranged from 100 to 150 in the combined spectra, depending on position in the echelle blaze. Spectra were normalized with a cubic spline that well fit the standard white dwarf LTT 9491. After normalization, individual spectra were combined. Lines of Fe II, Na I, and Al I selected for abundance determinations occur in 8 echelle orders drawn from both blue and red sides of MIKE. The equivalent widths were measured using the IRAF routine splot by fitting the profiles with a gaussian curve on the continuum-normalized spectra. In some cases, where blending was present, the blended profile was deconvolved using multiple gaussian profiles in order to

\footnotetext{
${ }^{5}$ See http://www.lco.cl/telescopes-information/magellan/instruments/mike/idl-tools
} 
extract the equivalent width of the line.

\section{Determination of Stellar Abundances}

Abundances of $\mathrm{Fe}, \mathrm{Al}$, and $\mathrm{Na}$ were derived from Magellan/MIKE spectra of the target stars. Following the precepts of Kraft and Ivans (2003), we focused on deriving Fe abundances from Fe II lines since this ion dominates in cool giants. The selection of Fe II lines and oscillator strengths was taken from Fulbright (2000). Of the 26 Fe II lines listed by Fulbright, we chose 10-13 to be measured in each star, aiming to find those near the center of the echelle blaze from each order where the signal-to-noise is highest.

The abundance analysis was restricted to lines with reduced equivalent width $\log (\mathrm{EW} / \lambda)<$ -4.5. In addition, we only used lines with $\mathrm{EW}>9 \mathrm{~m} \AA$, unless few stronger or no lines were available for a given species and star. Atomic parameters for all lines measured and individual equivalent widths are listed in Table 2.

We derived abundances by force-fitting equivalent widths in the 2010 version of MOOG (Sneden 19736). Effective temperatures for 11 of the stars were estimated using their $V-K$ colors and the calibration of Alonso et al. (1999). The K-magnitudes were taken from the 2MASS All Sky Catalog (Cutri et al. 2003) which lie within 0.005 mag of the TCS color system adopted by Alonso et al. (1999). One star did not have a $K$ magnitude; we used its $B-V$ color instead. A reddening of $E(B-V)=0.12$ mag was assumed (Harris 1996). These photometric estimates of effective temperature have small random errors: $0.01 \mathrm{mag}$ in $V-K$ corresponds to a change of only $10 \mathrm{~K} ; 0.01 \mathrm{mag}$ in $E(B-V)$ corresponds similarly to $30 \mathrm{~K}$. Systematic effects dominate, and are estimated at less than $100 \mathrm{~K}$.

Surface gravities were estimated using bolometric corrections from Alonso et al. (1999) for the effective temperatures already derived, a distance of $4.8 \pm 0.3 \mathrm{kpc}$ (van de Ven et al. 2006), and an assumed mass of $0.8 M_{\odot}$. The gravity is insensitive to reasonable uncertainties in mass and temperature as they enter the calculation only logarithmically. The largest source of error is the distance, which introduces a $1 \sigma$ random error of approximately 0.05 dex.

To obtain chemical abundances, Castelli \& Kurucz (2004) stellar atmosphere models were employed. These are one-dimensional, plane-parallel models without convective overshooting. We used $\alpha$-enhanced models $([\alpha / \mathrm{Fe}]=+0.4)$ that are generally appropriate for stars in a globular cluster. Models for the relevant parameters of each star were derived

\footnotetext{
${ }^{6}$ See http://www.as.utexas.edu/ ${ }^{\sim}$ chris/moog.html
} 
through interpolation. The effective temperature and surface gravity were fixed by the photometry as discussed above. We derived the metallicity $[\mathrm{Fe} / \mathrm{H}]$ and microturbulence $v_{t}$ iteratively. To minimize non-LTE effects, only Fe II lines were used. The microturbulence was determined in the usual manner by requiring that there be no trend of abundance with reduced equivalent width. The final adopted parameters for each star are given in Table 1.

Abundances for sodium and aluminum were derived using 2-4 weak lines per species and the stellar parameters estimated from the photometry and the Fe II lines. Four lines of Al I were selected: $\lambda 6696.03, \lambda 6698.67, \lambda 7835.32$, and $\lambda 7836.13$. The Na I analysis typically used 5 lines: $\lambda 4668.57$ (in some stars), $\lambda 5682.65, \lambda 5688.21, \lambda 6154.23$, and $\lambda 6160.75$. For one star, LEID 63027, we also included the Na resonance lines at $\lambda 5889.96$ and $\lambda 5895.94$ and they gave results consistent with those of weaker lines. Wavelengths of the transitions and their $g$-values were taken from the compilation of Fulbright (2000). The $g f$-values for Al came from Jacobson et al. (2009). For a subset of the stars, we could only derive upper limits to the abundance of aluminum. In these cases, the reported numbers are a median of the limits for the individual lines. Abundances are reported relative to solar values. The solar values in Asplund et al. (2005) were used for all elements except for iron, for which we assume $\log \epsilon(\mathrm{Fe})=7.52$ (Sneden et al. 1991). Fig. 4 shows a section from the MIKE spectra of two stars similar in $\mathrm{T}_{\text {eff }}, \log g$, and $[\mathrm{Fe} / \mathrm{H}]$ yet differing substantially in $\mathrm{Na}$ and helium.

After our analysis was completed, Johnson and Pilachowski (2010) published abundances for many red giants in Omega Cen based on HYDRA spectra of lower resolution $[\mathrm{R}(\lambda / \Delta \lambda)=18,000]$. Their sample includes nine of our targets. For the stars in common,

our values for $T_{\text {eff }}$ differ by a mean of $9 \mathrm{~K}$, and a maximum of $29 \mathrm{~K}$. The average difference in the values of $\log g$ amounts to 0.13. These authors derived an iron abundance generally based on a majority of Fe I lines. Some stars had no Fe II; others had one, or at most two lines from Fe II available. Fig. 5 shows the derived abundances for the stars in common where an mean offset of $-0.12 \pm 0.12$ dex exists between our values and those from Johnson $\&$ Pilachowski. The maximum deviation of 0.2 dex from this offset is less than the scatter of 0.65 dex among other values commonly found in the literature (see Fig. 5 of Johnson \& Pilachowski 2010).

\section{Results}

Helium was detected in 5 of the 12 red giants observed (see Fig. 2 and Table 1), and the equivalent widths of the lines differ from star to star. Considering the small range of effective temperature and luminosity in these giants, the equivalent width variation suggests a similar variation in the helium abundance. These helium lines, where detected, have larger 
equivalent widths than the helium lines found (Dupree et al. 2009) in metal-poor red giants with comparable temperatures in the field (Fig. 6). Additionally, where helium is not found, the lines in $\omega$ Cen tend to be weaker than in the field stars. For the $\omega$ Cen giants, no correlation is apparent between position in the color-magnitude diagram (CMD) and the presence of helium or with the iron abundance (Fig. 7). Our sample is small, and more targets are necessary to develop a clear pattern between helium line strength and the iron abundance, if, indeed it exists. In the metal-poor field giants, we also found no correlation of helium strength with $[\mathrm{Fe} / \mathrm{H}]$. On the subgiant branch of $\omega$ Cen also, Villanova et al. (2007) found poor correlation between $[\mathrm{Fe} / \mathrm{H}]$ and CMD location. The helium line, when present, may become stronger in the less luminous objects (Fig. 8).

\subsection{Overall Characteristics of the Presence of Helium}

The current view holds that increased helium abundance causes the blue main sequence of $\omega$ Cen (Norris 2004), and the gross characteristics of the $\omega$ Cen giants in the PHOENIX sample exhibiting helium are consistent with both the fraction and spatial distribution of the blue main sequence. The fraction of stars displaying helium, $42 \%$, is comparable to the fraction of blue main sequence stars (25 to 35\%) considered to have enhanced helium and also enhanced metals (Bedin et al. 2004; Piotto et al. 2005). The spatial distribution is also of interest. The blue main sequence stars have a higher spatial concentration near the cluster core than the red main sequence (Bellini et al. 2009; Sollima et al. 2007). Within the half-mass radius of the cluster $(\leq 4.2 \mathrm{arcmin})$, the ratio of blue to red main sequence stars ranges from 1.1 to 0.8 ; at 6 arcminutes from the center the ratio is still high at 0.5 ; further out from the center $(\geq 8$ arcmin) the ratio falls to 0.4 . Taking the center of the $\omega$ Cen cluster (Anderson \& van der Marel 2010) as RA $=13: 26: 47.24$, Dec $=-47: 28: 46.45$ (J2000.0), our sample of twelve giants is evenly split in radius at a distance of 6 arcminutes from the center. Of the 6 stars within 6 arcminutes from the center, 3 have helium or 50\%; this fraction falls to $33 \%$ (2 out of 6 targets) for the more distant objects. While our sample is small, these fractions are consistent with the spatial behavior of the blue:red ratio. Thus, the percentage detection and the spatial distribution of the helium detections are consistent with the blue main sequence population.

The equivalent widths of the detected helium lines are stronger than the upper limits of the non-detections by factors of 3 to 10. Judging from our calculations for the quiet Sun, the amount of this enhancement could correspond to a change in helium abundance by more than a factor of two. However, conditions in a red giant chromosphere are certainly different from a dwarf star. Outflows can increase the absorption from the metastable lines in helium 
(Dupree et al. 2009). Variability in the chromospheric structure could occur also, although it is not likely to be large judging by the lack of $\mathrm{H}-\alpha$ wing emission, and the weak Ca II emission reversals in the optical spectra of these giants. Detailed non-LTE semi-empirical calculations are underway to extract the helium abundance, and the chromospheric models must be constrained by $\mathrm{H}-\alpha$ and Ca II line profiles.

\subsection{Abundances of the Giant Stars}

The relation between the abundance of iron $[\mathrm{Fe} / \mathrm{H}]$ and the presence of helium is shown in Fig. 9. Stars with $[\mathrm{Fe} / \mathrm{H}]<-1.8$ do not exhibit helium; the majority of stars in the range $-1.8 \leq[\mathrm{Fe} / \mathrm{H}] \leq-1.4$ display the helium transition, and the one metal-rich star in our sample at $[\mathrm{Fe} / \mathrm{H}]=-1.15$ does not show helium 7 On the main sequence, the metallicity, $[\mathrm{M} / \mathrm{H}]$ corresponds to -1.26 for the blue main sequence and -1.57 for the red main sequence (Piotto et al. 2005). An empirical transformation from $[\mathrm{M} / \mathrm{H}]$ to $[\mathrm{Fe} / \mathrm{H}]$ was estimated by Villanova et al. (2007) from a comparison of subgiant spectra to spectral syntheses for various values of $[\mathrm{M} / \mathrm{H}]$. The best-fitting synthesis for $[\mathrm{M} / \mathrm{H}]$ was compared to values of $[\mathrm{Fe} / \mathrm{H}]$ for many subgiants in $\omega$ Cen. The correction, obtained from the mean difference between $[\mathrm{M} / \mathrm{H}]$ and $[\mathrm{Fe} / \mathrm{H}]$ amounts to -0.11 dex applied to values of $[\mathrm{M} / \mathrm{H}]$. For the blue and red main sequences, this leads to $[\mathrm{Fe} / \mathrm{H}]=-1.37$ and -1.68 respectively. An additional minor red branch can be found on the main sequence (Villanova et al 2007; Anderson and van der Marel 2010) termed MS-a which has higher $[\mathrm{Fe} / \mathrm{H}](-1.1$ to -0.6$)$ than the blue main sequence. We do not find a correlation between the $[\mathrm{Fe} / \mathrm{H}]$ distribution and the presence of the He I $\lambda 10830$ line.

A different indication of processed material can be found in the light element abundances of $\mathrm{Na}$ and $\mathrm{Al}$ which signal high temperature $\mathrm{H}$ burning via the CNO cycle (Denisenkov \& Denisenkova 1989; Langer et al. 1993) in a previous stellar generation (Gratton et al. 2001). In red giants, the outer convective envelope will never reach the the H-burning shell where the light elements are produced during evolution on the red giant branch (Gratton et al. 2004). For enhanced light elements to be present, they must have originated in a previous generation of stars. The abundances of $[\mathrm{Na} / \mathrm{Fe}]$ and $[\mathrm{Al} / \mathrm{Fe}]$ are correlated with each other, and clearly enhanced in the stars displaying He I lines (Fig. 10). Stars without a helium detection have low values of $[\mathrm{Na} / \mathrm{Fe}]$ and principally upper limits on the $[\mathrm{Al} / \mathrm{Fe}]$ abundance. These enhancements of $\mathrm{Na}$ and $\mathrm{Al}$ agree with values from red giants in $\omega$ Cen (Norris \& Da

\footnotetext{
${ }^{7}$ Our abundance for LEID 54084, $[\mathrm{Fe} / \mathrm{H}]=-1.79$, differs from the Johnson \& Pilchowski (2010) value of $[\mathrm{Fe} / \mathrm{H}]=-1.5$. Such a shift would not significantly change the distribution of helium detections.
} 
Costa 1995; Johnson \& Pilachowski 2010). And the values of $[\mathrm{Na} / \mathrm{Fe}]$ and $[\mathrm{Al} / \mathrm{Fe}]$ for the helium and non-helium giants are similar to the abundances found in NGC 2808 for two stars, one each from the blue and red components of the main sequence respectively (Bragaglia et al. 2010a). Thus the red giants in $\omega$ Cen exhibiting the abundance pattern expected from the high temperature H-burning processes are the majority of red giants displaying enhanced He lines.

\subsection{Evolutionary Connections}

The population structure in $\omega$ Cen is quite complex. We now focus on the spectroscopic $[\mathrm{Fe} / \mathrm{H}]$ values, and consider how the helium detections fit in with the continuity of the colormagnitude diagram (CMD) and the various population components. We follow the ideas of Villanova et al. (2007), to interpret the presence of helium in the $\omega$ Cen giants and diagram these ideas in Fig. 11. These authors evaluated three factors in presenting an evolution scenario: (1) morphological continuity on the CMD; (2) population fraction; and (3) spectroscopic metallicity of the stars. The bifurcated main sequence (MS) principally represents two values of $[\mathrm{Fe} / \mathrm{H}],-1.68$ (spanning $0.2 \mathrm{dex}$ ) and -1.37 , and a minority population with $[\mathrm{Fe} / \mathrm{H}]=-1.1$ to -0.6 . Detailed abundance study of the subgiant branch (SGB) reveals (Villanova et al. 2007) four or five components which peak at specific values of $[\mathrm{Fe} / \mathrm{H}]$ and the abundances typically span 0.3 to 0.6 dex (FWHM) each, and range in $[\mathrm{Fe} / \mathrm{H}]$ from -1.7 to -0.6 . These too are marked in Fig. 11 and denoted by SGB: A, B, C, and D. Possibly an additional component bridges the abundance range between $\mathrm{B}, \mathrm{C}$ and $\mathrm{D}$. On the red giant branch (RGB), Johnson \& Pilachowski (2010) identified 4 components which they denote (following Sollima et al. 2005), RGB: MP, MInt1, MInt 2+3 and RGB-a where MP and MI stands for metal-poor and metal-intermediate. The abbreviation RGB-a denotes the anomalous red giant branch (Lee et al. 1999; Pancino et al. 2000). To understand the evolutionary relationships among these components, Villanova et al. (2007) suggest 'cross-over' occurs from the red main sequence (rms) to the subgiant (SGB) components A, B, and C, and from the blue main sequence (bms) to the subgiant subpopulations $\mathrm{B}$ and $\mathrm{C}$, and perhaps the intermediate component located between $\mathrm{B}, \mathrm{C}$, and $\mathrm{D}$.

From the subgiant branch to the red giant branch, component SGB-A feeds into the RGB-MP, and stars on SGB-D move to RGB-a. Thus the absence of helium in giants for

$[\mathrm{Fe} / \mathrm{H}]<-1.8$ is not surprising following the route from the MS-rms to RGB-MP. The MS-rms also overlaps the metallicity of SGB-B,C and the component percentages suggest that MS-rms evolves to SGB-B,C, and then to RGB-MInt1 (Villanova et al. 2007), The $\omega$ Cen stars with helium span $[\mathrm{Fe} / \mathrm{H}]=-1.8$ to -1.45 in our sample. Villanova et al. (2007) 
conclude, based on photometric and spectroscopic information that the MS-bms evolves through SGB-B,C which then, based on the metallicity overlap, contributes to RGB-MP and RGB-MInt1, and perhaps RGB-MInt2+3. In their abundance study of 855 red giants in $\omega$ Cen, Johnson and Pilachowski (2010) found that O-poor, Na/Al rich stars occur in three out of the 4 populations of red giants in the cluster, but not in the RGB-a cohort. The intermediate population of the [O/Fe] poor giants (RGB-MInt1 and RGB-MInt 2+3) shares attributes with the blue main sequence: spatial distribution, metallicity, and percentages, leading Johnson \& Pilachowski (2010) to suggest that the [O/Fe] depletion (coupled with the $\mathrm{Na} / \mathrm{Al}$ enhancement) signals those giants whose progenitors belonged to the blue main sequence. The mix of helium detections and non-detections between -1.8 and -1.45 appears consistent with the crossovers outlined above.

We are left with an apparently anomalous object, LEID 38269, which is metal-rich, $[\mathrm{Fe} / \mathrm{H}]=-1.16$ and does not show helium. Its evolutionary path currently places it on the RGB-MInt2+3, and its metallicity would seem to prevent an origin on the metal-poor MS-rms where helium is weak or absent. However, age dating of the subgiant population in $\omega$ Cen provides a clue to this object.

In addition to continuity of metallicity values, Villanova et al. (2007) note that the subgiant branch offers an optimum region of the CMD to determine ages by comparison to stellar isochrones. Interestingly, they find the broadest dispersion in stellar ages for stars with $-1.8 \leq[\mathrm{Fe} / \mathrm{H}] \leq-1.5$ which echoes earlier results from studies of the main sequence turnoff stars by Stanford et al. (2006). Over this metallicity range, the estimated ages span almost a factor of two, from 0.6 of the age of the oldest stars continuously ranging to the oldest stars in the cluster. Thus the mixed appearance of helium in our targets at this metallicity range appears in harmony with the span of ages found in subgiants. At the highest iron abundance $([\mathrm{Fe} / \mathrm{H}] \sim-1.1)$, three subgiants in the Villanova et al. (2007) sample have ages commensurate with those of the most metal-poor stars suggesting a simple age-metallicity correlation does not exist in $\omega$ Cen. This might occur as a result of several star-forming episodes. Material from Type II supernovae creates one stellar generation which is followed by the polluting effects from longer-lived metal-poor intermediate-mass asymptotic giant branch stars on a subsequent stellar generation (Johnson \& Pilachowski 2010). LEID 38269 also does not show enhanced $\mathrm{Na}$ and $\mathrm{Al}$, and would plausibly belong to this older population. It is the coolest and faintest star in our sample, and could form a part of the 'anomalous' population in $\omega$ Cen. 


\subsection{Concluding Comments}

The direct detection of a helium abundance variation and its correlations with both $[\mathrm{Fe} / \mathrm{H}]$ and light element abundances of $\mathrm{Al}$ and $\mathrm{Na}$ in this small sample of red giants, supports the conjecture that helium is enhanced in the blue main sequence component of the $\omega$ Cen cluster. It appears likely that the helium-enhanced stars contain the products of high-temperature hydrogen burning produced by an earlier generation of stars. Spectra of additional targets would be useful to thoroughly understand the relationship between $[\mathrm{Fe} / \mathrm{H}]$ and the presence of helium.

The infrared helium line at $10830 \AA$ appears to be a powerful probe of the presence of helium in warm giant stars. Detailed non-LTE studies of its formation under a variety of conditions are needed to be able to extend the interpretation to a larger range of temperatures and luminosities, and also to extract the absolute abundance of helium.

This research has made use of NASA's Astrophysics Data System. We thank the US Gemini Office for assistance in preparing Phase II, and providing travel support to GeminiS. We thank Steve Margheim and Claudia Winge for Phase II advice and support during our classical run at Gemini-S. JS acknowledges support provided by NASA through Hubble Fellowship grant HST-HF-51237.01, awarded by the Space Telescope Science Institute, which is operated by the Association of Universities for Research in Astronomy, Inc., for NASA, under contract NAS 5-26555.

Facilities: Gemini:South (PHOENIX), Magellan/Clay (MIKE)

\section{REFERENCES}

Alonso, A., Arribas, S., \& Martínez-Roger, C. 1999, A\&AS, 140, 261 (Erratum: 2001, A\&A, $376,1039)$

Anderson, J. 1997, Ph.D. thesis, Univ. California, Berkeley.

Anderson, J., \& van der Marel, R. P. 2010, ApJ, 710, 1032

Asplund, M., Grevesse, N., \& Sauval, A. J. 2005, in ASP Conf. Ser. 336, T. G. Barnes III, \& F. N. Bash eds., (San Francisco: ASP), p. 25

Avrett, E. H., \& Loeser, R. 2008, ApJS, 175, 229

Bedin, L. R., Piotto, G., Anderson, J., Cassisi, S., King, I. R., Momany, Y., \& Carraro, G 2004, ApJ, 605, L125 
Behr, B. B., Cohen, J. G., McCarthy, J. K., \& Djorgovski, S. G. 1999, ApJ, 517, L135

Bellini, A., Bedin, L., R., Piotto, G., Milone, A. P., Marino, A. F., \& Villanova, S. 2010, AJ, 140, 631

Bellini, A., Piotto, G., Bedin, L. R., King, I. R., Anderson, J., Milone, A. P., \& Momany, Y. 2009, A\&A, 507, 1393

Bernstein, R. A., Shectman, S. A., Gunnels, S. M., Mochnacki, S., \& Athey, A. E. 2003, Proc. SPIE, 4841, 1694

Bragaglia, A. et al. 2010a, ApJ, 720, L41

Bragaglia, A., Carretta, E., Gratton, R., D’Orazi, V., Cassisi, S., \& Lucatello, S. 2010b, A\&A, 519, A60

Breckinridge, J. B., \& Hall, D. N. B. 1973, Sol. Phys., 28, 15

Castelli, F., \& Kurucz, R. L., 2004, A\&A, 419, 725

Catelan, M. 2009, in IAU Symp. 258, E. E. Mamajek, D. R. Soderblom, \& R. F. G. Wyse, eds, (Cambridge: Cambridge U. Press), p. 209

Choi, E., \& Yi, S. K. 2008, MNRAS, 386, 1332

Cutri, R. M. et al. 2003, The IRSA 2MASS All-Sky Point Source Catalog, NASA/IPAC Infrared Science Archive (CDA/ADC Collection of Electronic Catalogues, 2246)

Da Costa, G. S., Norris, J., \& Villumsen, J. V. 1986, ApJ, 308, 743

D’Antona, F., \& Caloi, V. 2004, ApJ, 611, 871

D'Antona, F., \& Caloi, V. 2008, MNRAS, 390, 693

D’Cruz, N. L., et al. 2000, ApJ, 530, 352

Denisenkov, P. A., \& Denisenkova, S. N. 1989, A. Tsir., 1538, 11

Dupree, A. K., Sasselov, D. D., \& Lester, J. B. 1992, ApJ, 387, L85

Dupree, A. K., Smith, G. H., \& Strader, J. 2009, AJ, 138, 1485

Freeman, K. C., \& Rodgers, A. W. 1975, ApJ, 201, L71

Fulbright, J. P. 2000, AJ, 120, 1841 
Geyer, E. H. 1967, Z. fur Ap., 66, 16

Gratton, R. G. et al. 2001, A\&A, 369, 87

Gratton, R. G., Sneden, C., \& Carretta, E. 2004, ARA\&A, 42, 385

Gratton, R. G., Carretta, E., Bragaglia, A., Lucatello, S., \& D’Orazi, V. 2010, A\&A, 517, A81

Harris, W. E. 1996, AJ, 112, 1487

Hinkle, K. H. et al. 2003, Proc. SPIE, 4834, 353

Jacobson, H. R., Friel, E. D., \& Pilachowski, C. A. 2009, AJ, 137, 4753

Johnson, C. I., \& Pilachowski, C. A. 2010, ApJ, 722, 1373

Kraft, R. P., \& Ivans, I. I. 2003, PASP, 115, 143

Langer, G. E., Hoffman, R., \& Sneden, C. 1993, PASP, 105, 301

Lee., Y.-W., Joo, J.-M., Sohn, Y.-J., Rey, S.-C., Lee, J.-C., \& Walker, A. R. 1999, Nature, 402, 55

Lub, J. 2002, ASP Conf. Ser., F. van Leeuwen J. D. Hughes, \& G. Piotto, eds, (San Francisco: ASP), 265, 95 .

Momany, Y., Bedin, L. R., Cassisi, S., Piotto, G., Ortolani, S., Recio-Blanco, A., De Angeli, G., \& Castelli, F. 2004, A\&A, 420, 605

Norris, J. E. 2004, ApJ, 612, L25

Norris, J. E., \& DaCosta, G. S. 1995, ApJ, 441, L81

Pancino, E., Ferraro, F. R., Bellazzini, M., Piotto, G., \& Zoccali, M. 2000, ApJ, 534, L83

Piotto, G. et al. 2005, ApJ, 621, 777

Piotto, G. et al. 2007, ApJ, 661, L53

Piotto, G. 2009, in IAU Symposium 258, E. E. Mamajek, D. R. Soderblom, \& R. F. G. Wyse, eds, (Cambridge: Cambridge U. Press), p. 233

Reijns, R. A., Seitzer, P., Arnold, R., Freeman, K. C., Ingerson, T.,van den Bosch, R. C. E., van de Ven, G., \& de Zeeuw, P. T. 2006, A\&A, 445, 503 
Smith, G. H., Dupree, A. K., \& Strader, J. 2004, PASP, 116, 819

Sneden, C. 1973, Ph.D. thesis, U. Texas at Austin, Dissertation Abstracts International, Vol. 35-01, Section B, p. 28

Sneden, C., Kraft, R. P., Prosser, C. F., \& Langer, G. E. 1991, AJ, 102, 2001

Sollima, A., Ferraro, F. R., Bellazzini, M., Origlia, L., Straniero, O., \& Pancino, E. 2007, ApJ, 654, 915

Sollima, A., Ferraro, F. R., Pancino, E., \& Bellazzini, M. 2005, MNRAS, 357, 265

Stanford, L. M., Da Costa, G. S., Norris, J. E., \& Cannon, R. D. 2006, ApJ, 647, 1075

van de Ven, G., van den Bosch, R. C. E., Verolme, E. K., \& de Zeeuw, P. T. 2006, A\&A, 445,513

van Dokkum, P. G. 2001, PASP, 113, 1420

van Leeuwen, F., Le Poole, R. S., Reijns, R. A., Freeman, K. C., \& de Zeeuw, P. T. 2000, A\&A, 360, 472

Villanova, S. et al. 2007, ApJ, 663, 296

Woolley, R. v. d. R. 1966, Royal Obs. Ann, 2, 1 


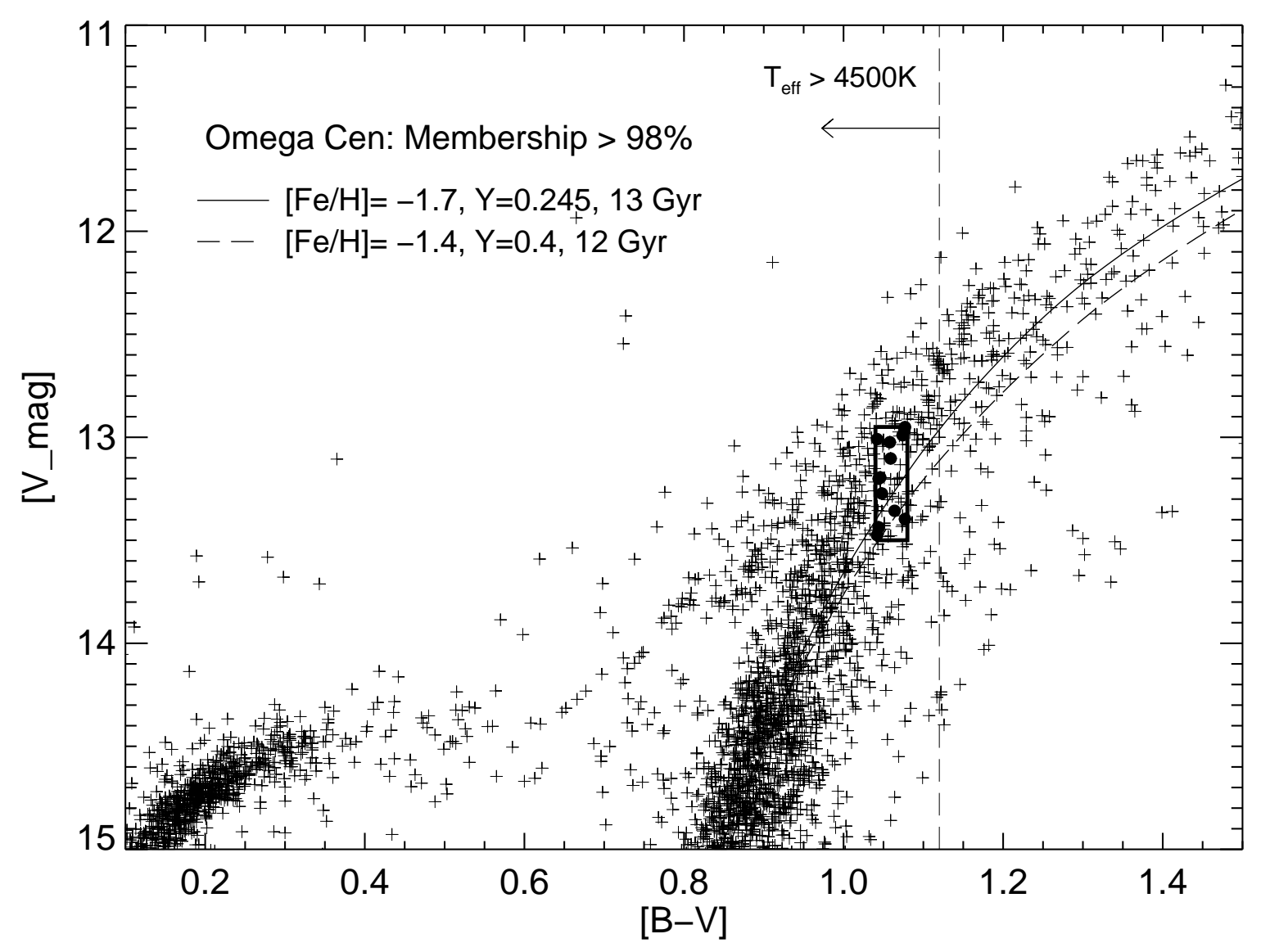

Fig. 1. - Color-magnitude diagram of $\omega$ Cen stars from van Leeuwen et al. (2000) with better than $98 \%$ probability of membership. Filled circles represent the stars observed. Note these targets are stars on the red giant branch; the target selection purposely avoids asymptotic branch objects. Isochrones are taken from the Dartmouth Stellar Evolution Database (http://stellar.dartmouth.edu/ models/) for the two majority populations in Omega Cen (Norris 2004;Piotto et al. 2005) and taking $\mathrm{E}(\mathrm{B}-\mathrm{V})=0.11$ (Lub 2002). 

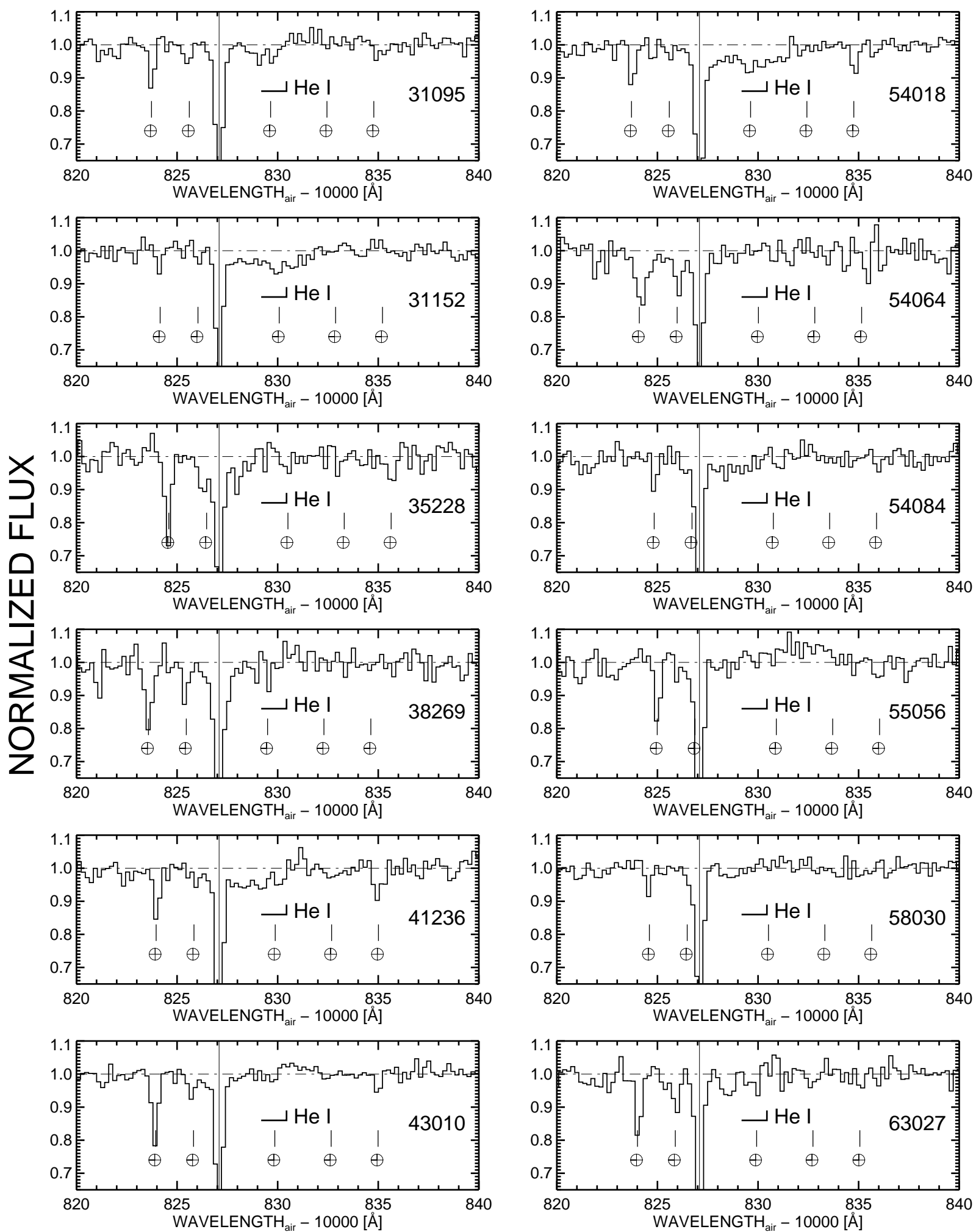

Fig. 2.- Spectra from PHOENIX (binned to a resolution element) are aligned on the rest wavelength of the photospheric line of Si I at $\lambda$ 10827.09. The LEID identification numbers are noted in each panel. The position of the strongest helium line component is marked by a vertical bar with an extent to shorter wavelengths for the weak components. Water vapor lines are noted by the $\oplus$ symbol. 


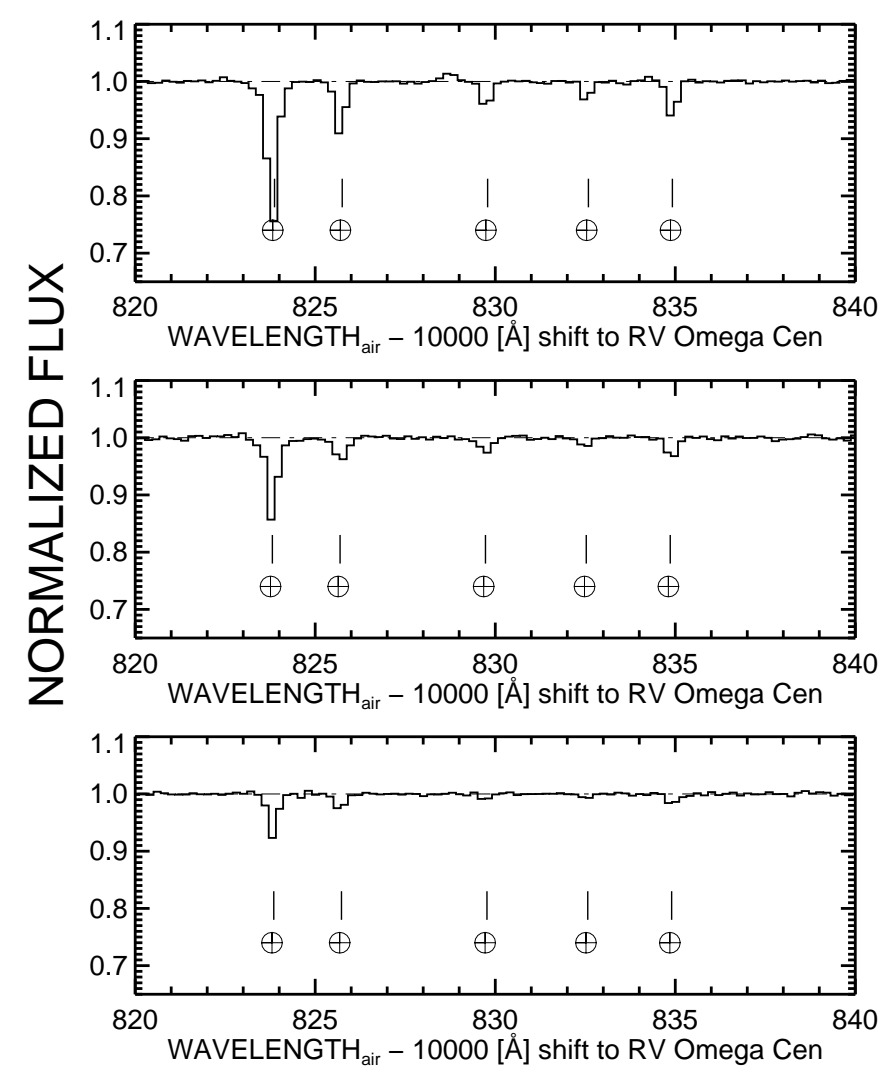

Fig. 3.- Spectra of HR 5231, a fast rotating B2.5IV star showing the variation of the strengths of the telluric water line. These spectra have been shifted by $-230 \mathrm{~km} \mathrm{~s}^{-1}(8.3 \AA)$ to correspond to the average radial velocity of our target stars, and to make comparison easier with Fig. 2. The observed strength of the water vapor line near the helium feature (the central water vapor line near $\lambda 10830$ in this figure) ranged from 4 to $18 \mathrm{~m} \AA$ in the target stars. Wavelengths are taken from Breckenridge \& Hall (1973). 


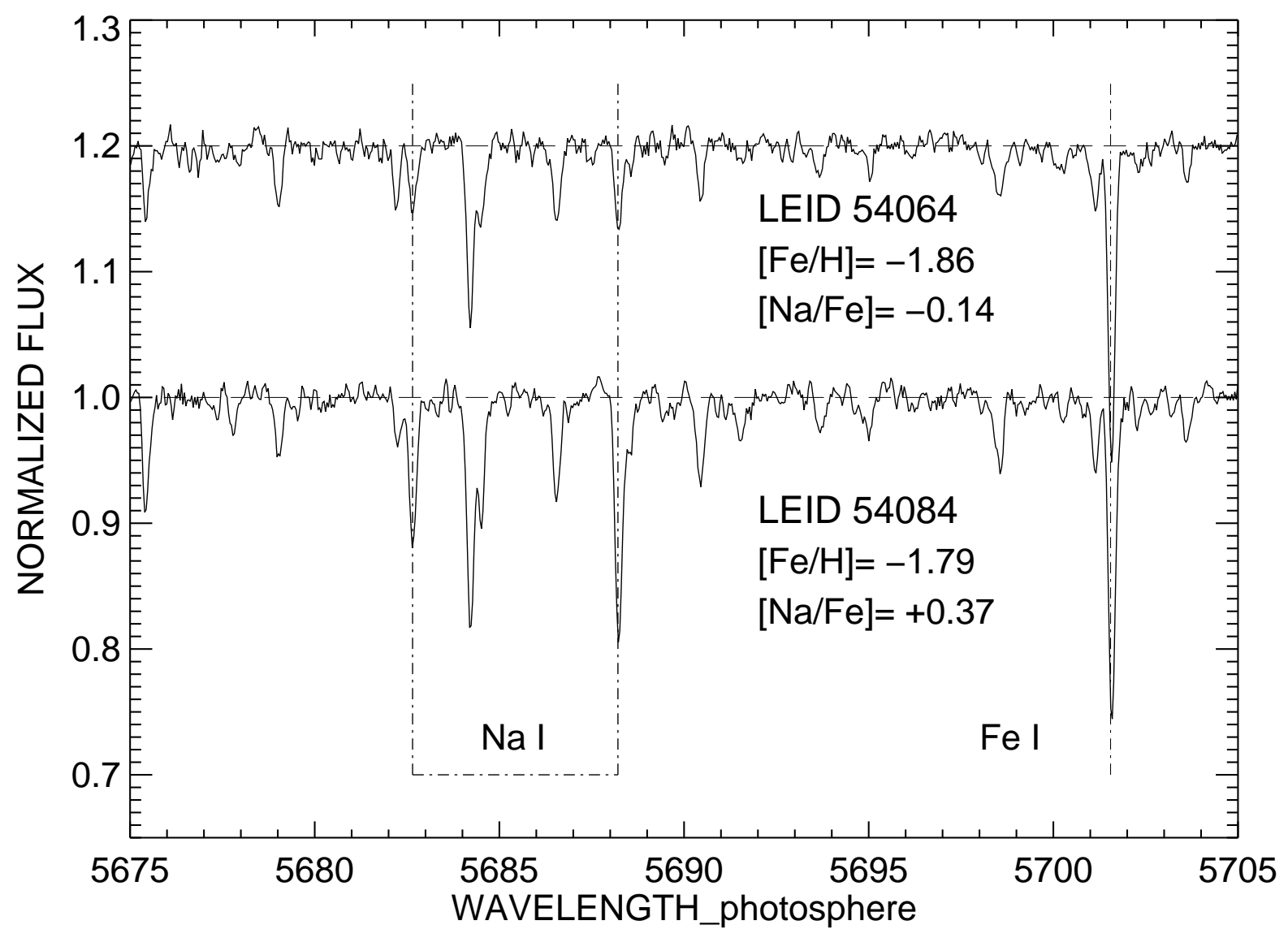

Fig. 4.- Region of the Na lines (laboratory wavelengths) in MIKE spectra of two stars: LEID 54064 and LEID 54084. They have similar values of $[\mathrm{Fe} / \mathrm{H}]$, equivalent $T_{\text {eff }}$ and $\log g$, and enhanced $\mathrm{Na}$ in LEID 54084, but not in LEID 54064. LEID 54084 shows helium; LEID 54064 does not. The spectrum of LEID 54064 has been offset by +0.2 in the normalized flux. 


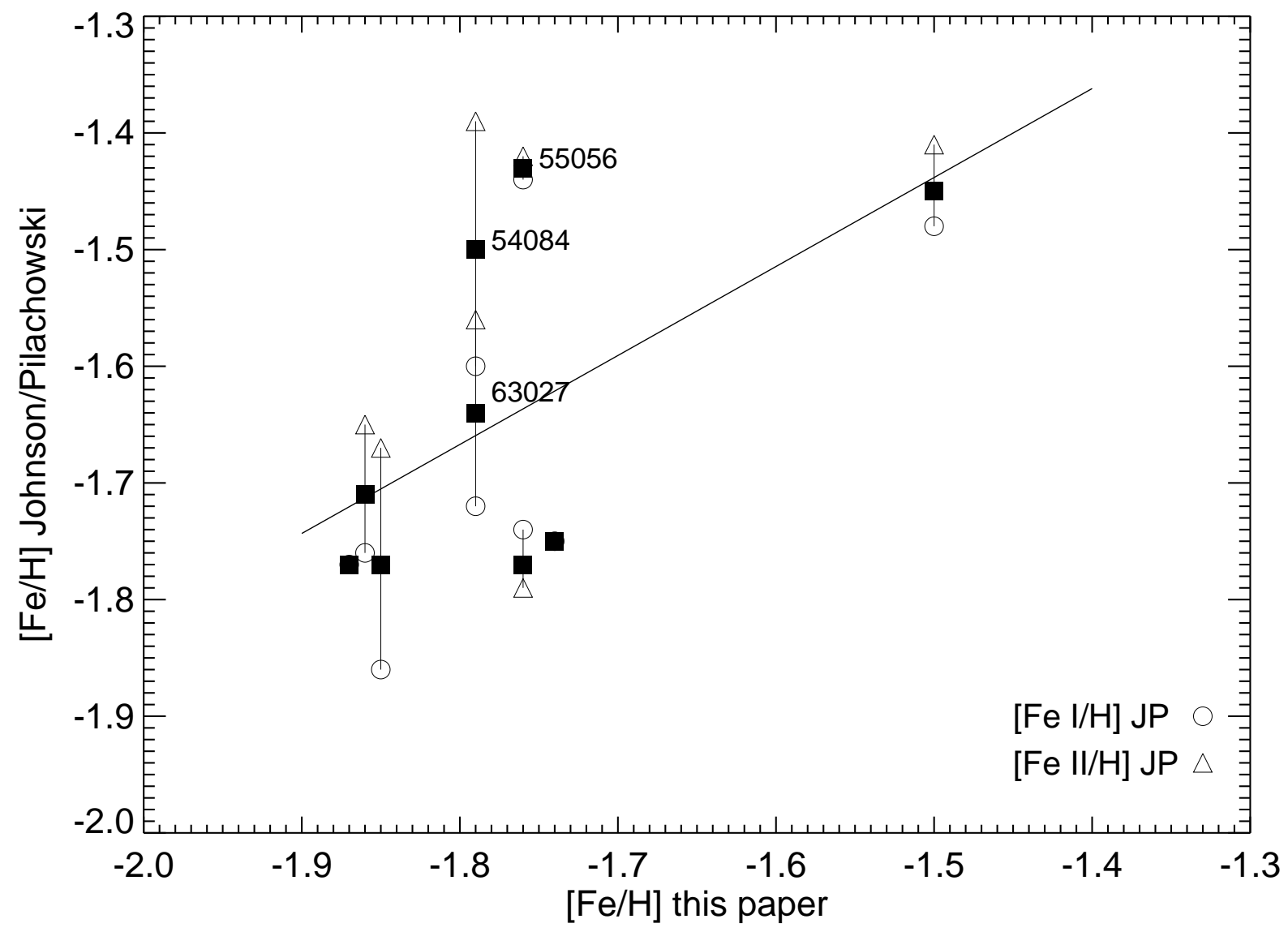

Fig. 5.- Comparison of Johnson \& Pilachowski (2010) $[\mathrm{Fe} / \mathrm{H}]$ abundances from Fe I $(\bigcirc$ symbol) and, in some cases one line or two of Fe II $(\triangle$ symbol), and the average value ( symbol) with the abundances derived here from Fe II lines in MIKE/Magellan echelle spectra. The straight line marks a linear relation least-squares fit where the mean difference between determinations amounts to $\sim-0.12 \pm 0.12$ dex. 


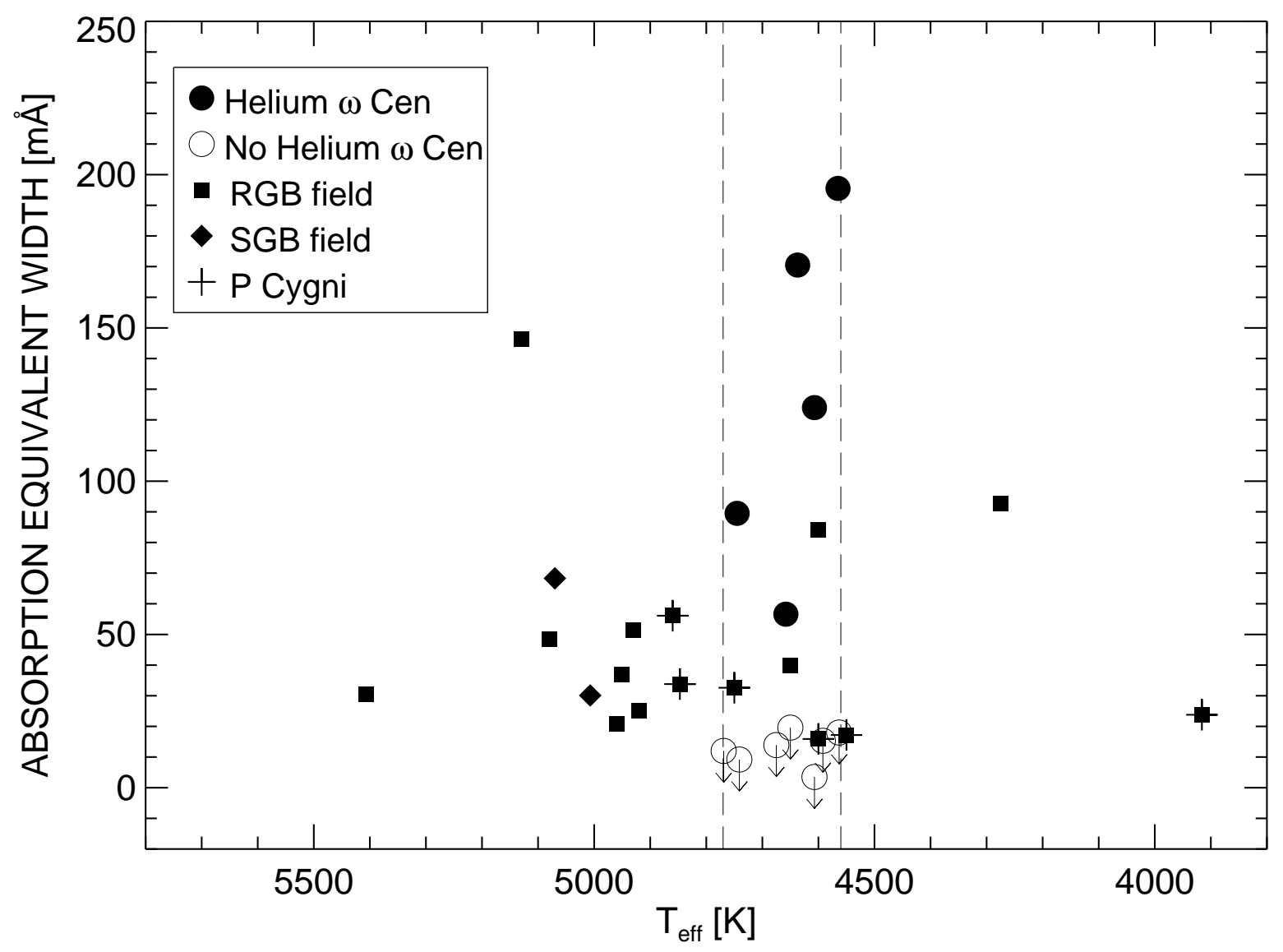

Fig. 6.- Helium equivalent width measures for the $\omega$ Cen giants (this paper), and metalpoor red giants and subgiants in the field (from Dupree et al. 2009). The broken vertical lines mark the narrow temperature region of the $\omega$ Cen sample. 

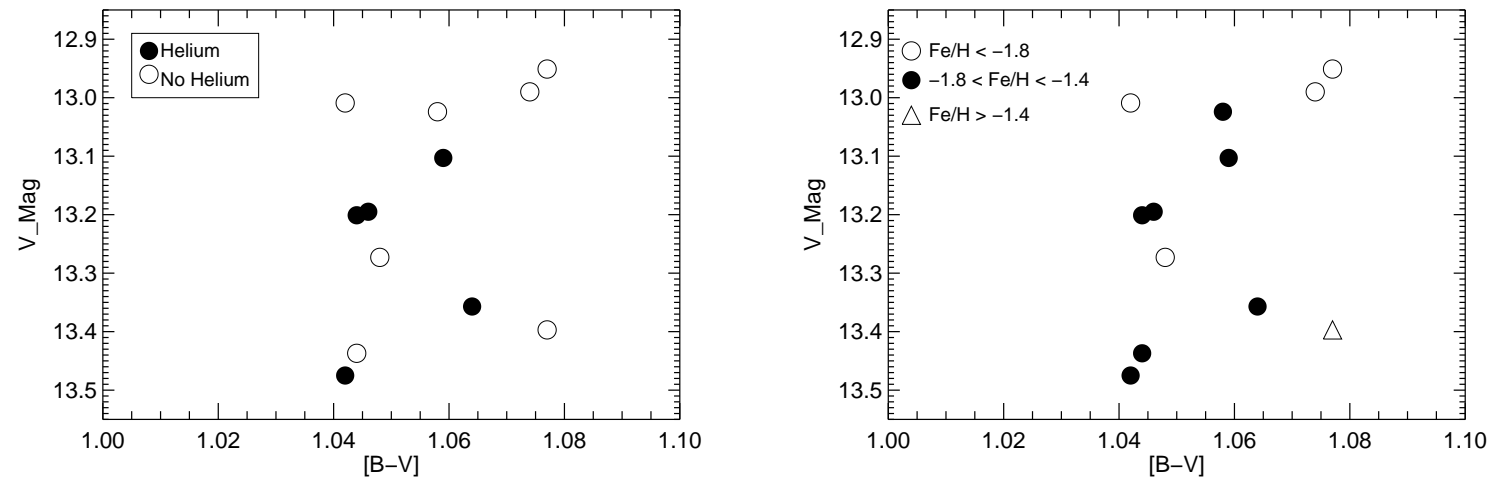

Fig. 7.- The color-magnitude diagram for the $\omega$ Cen stars observed with PHOENIX. In the left panel, the stars are labeled according to the presence of a He I line in the spectra. In the right panel, stars are labeled according to $[\mathrm{Fe} / \mathrm{H}]$ abundance. 


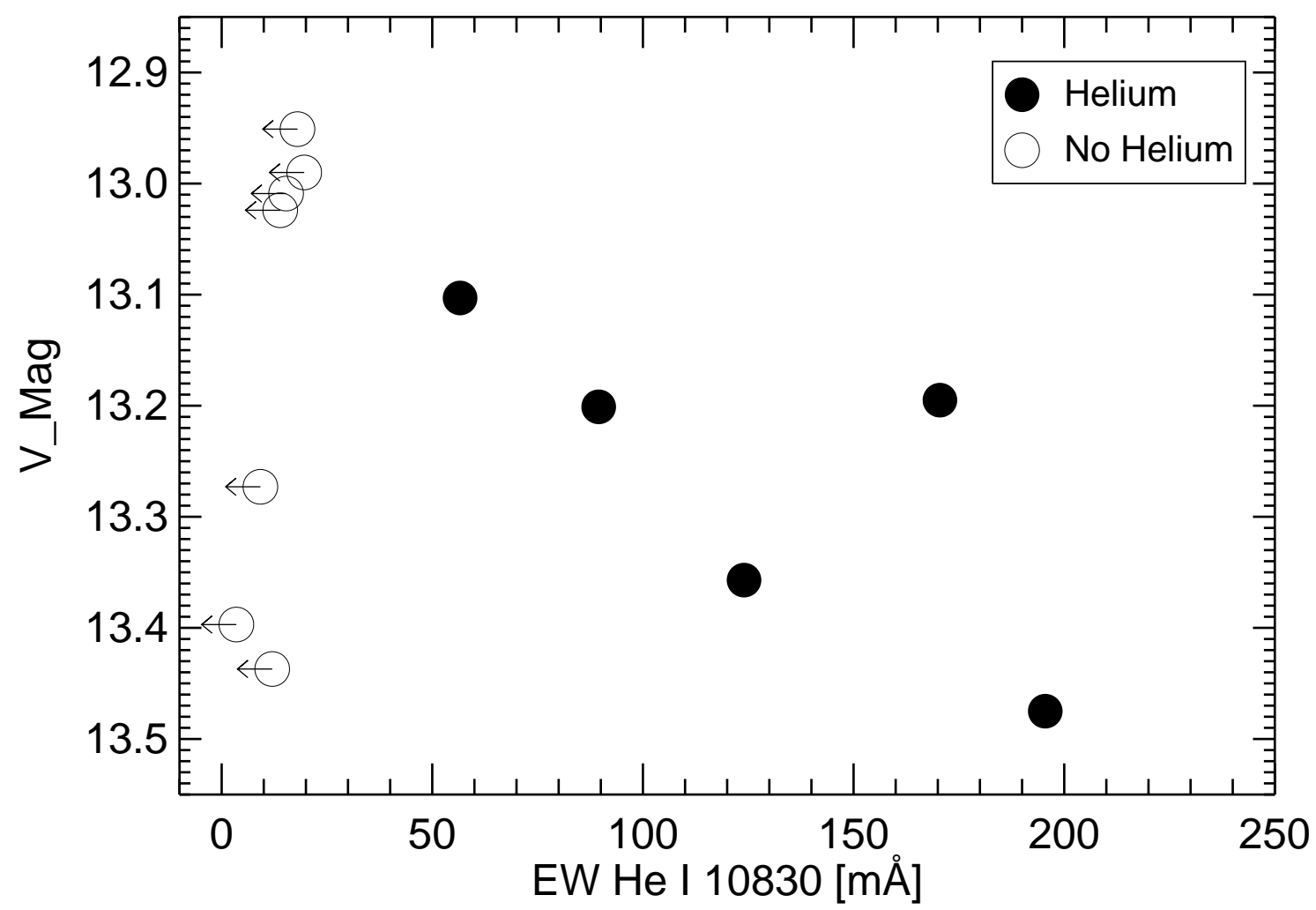

Fig. 8.- Relation between the helium equivalent width and apparent magnitude of our targets suggests the line may become stronger in the less luminous stars. The non-detections can be considered upper limits to the helium equivalent widths. 


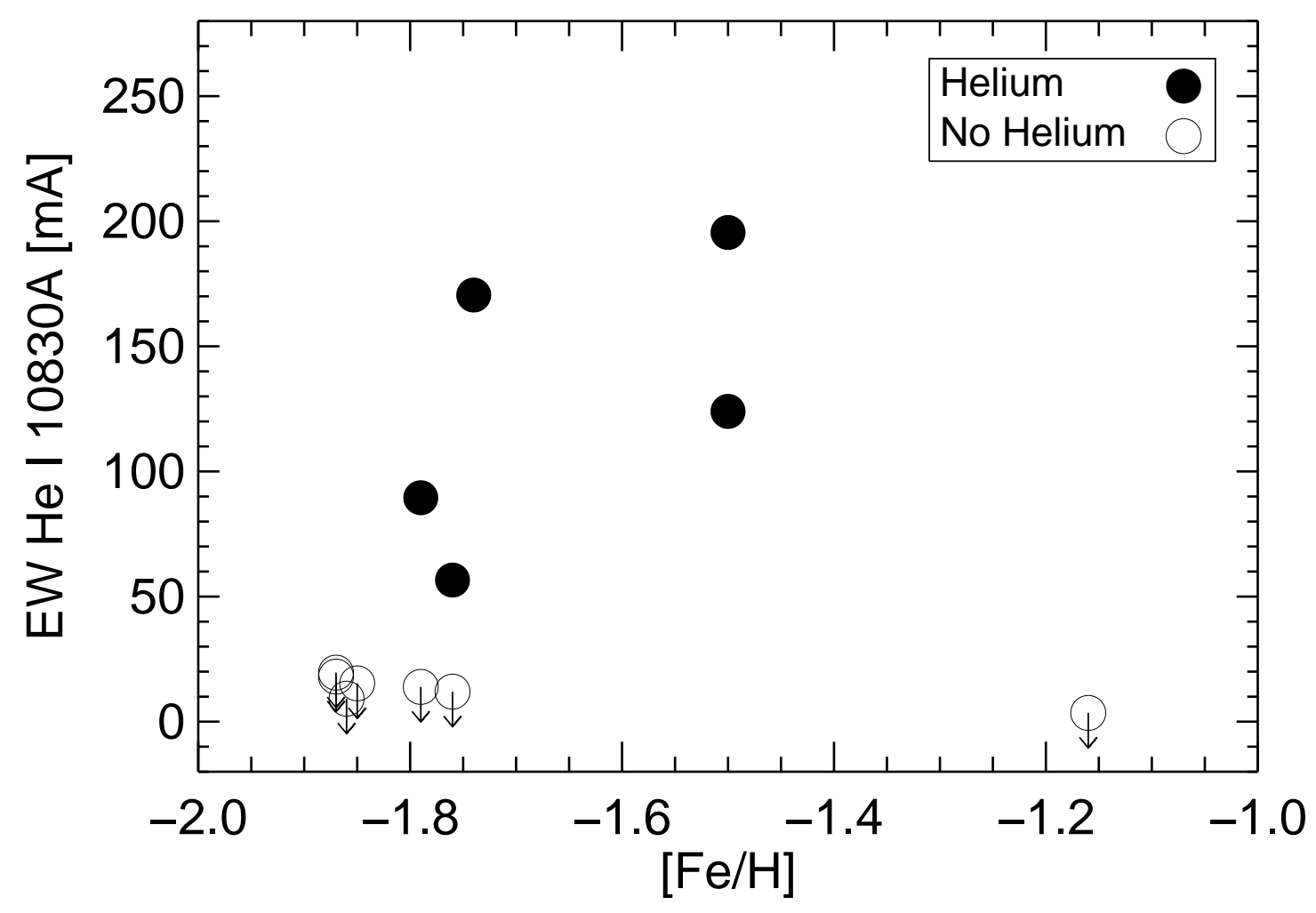

Fig. 9.- Dependence of equivalent width of the helium line on the value of $[\mathrm{Fe} / \mathrm{H}]$. The equivalent widths for the non-detections, marked as upper limits, represent the widths of the water vapor lines near the wavelength of helium in the target stars. 


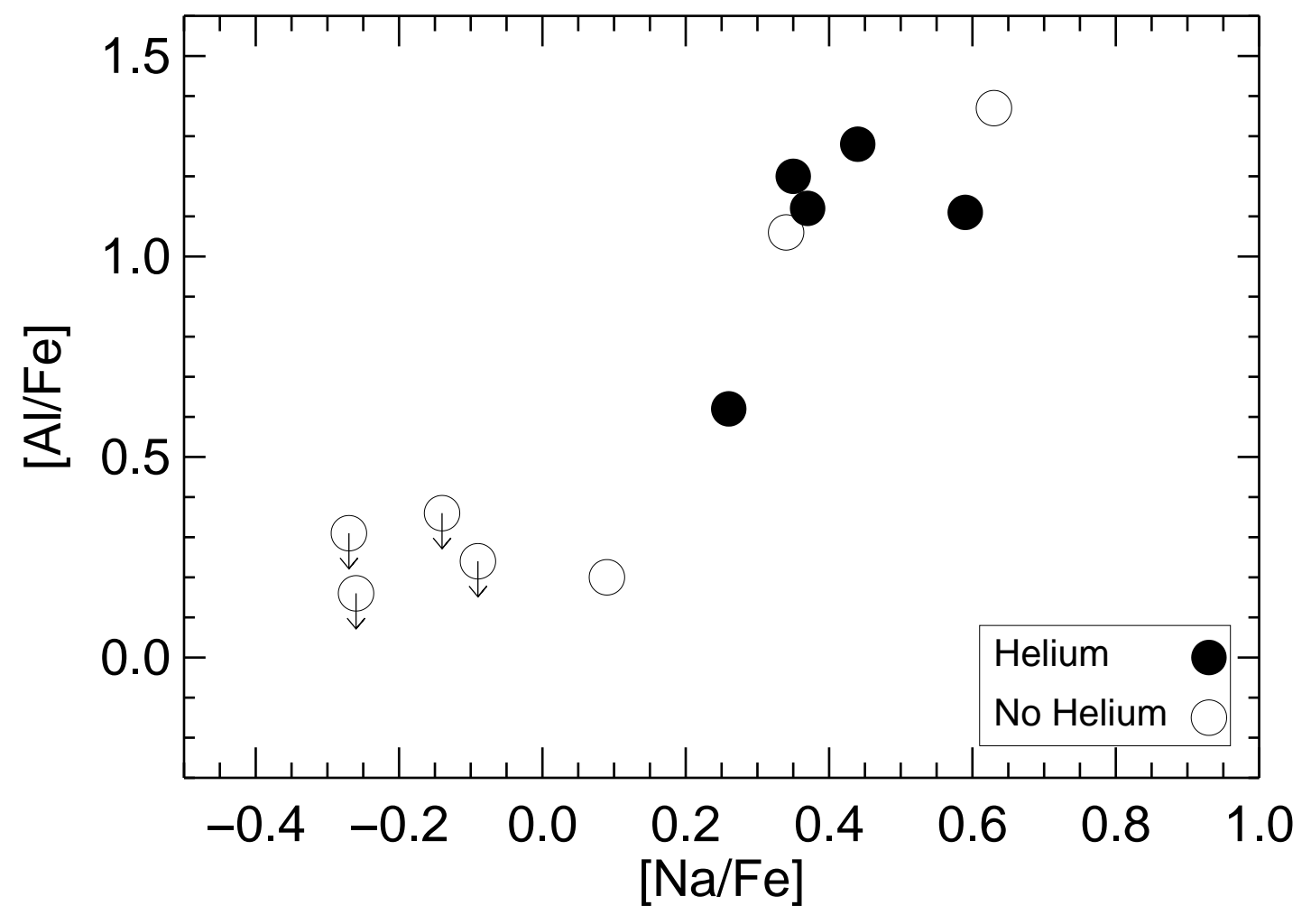

Fig. 10.- Relation between $[\mathrm{Al} / \mathrm{Fe}]$ and $[\mathrm{Na} / \mathrm{Fe}]$ where the stars with detected helium are preponderantly those with enhanced $\mathrm{Al}$ and $\mathrm{Na}$. 


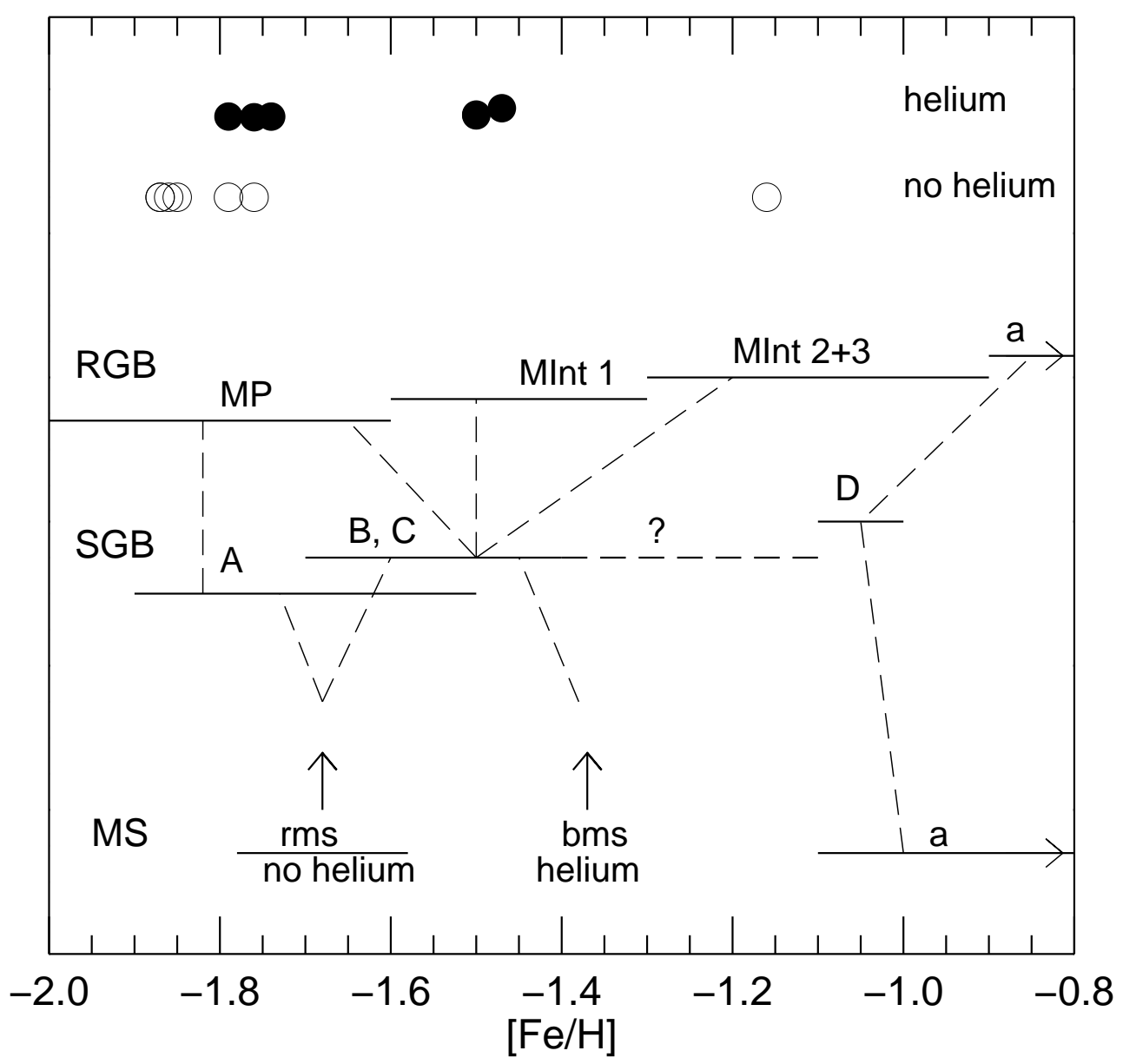

Fig. 11.- A posited evolutionary sequence of the populations in $\omega$ Cen in relation to the presence or not of helium in the red giants reported here (indicated by open or filled circles). The $[\mathrm{Fe} / \mathrm{H}]$ abundances of population components for the main sequence (Piotto et al. 2005), the sub-giant branch, SGB (Villanova et al. 2007) and the red giant branch RGB, (Johnson \& Pilachowski 2010) are marked. 'Cross-over' among the components, has been suggested by Villanova et al (2007) as possibly occurring from the red main sequence (rms) to the subgiant (SGB) components A, B, and C as well as from the blue main sequence (bms) to the subgiant subpopulations B and C. Johnson \& Pilachowski's results (2010) also support the bms as the progenitor of the MInt1 and MInt2+3 RGB population. Ages are not considered here; the estimate by Villanova et al. (2007) suggests that the stars without a helium detection belong to the oldest stellar generation in $\omega$ Cen (see text). 
Table 1. Omega Cen Giants Observed

\begin{tabular}{ccccccccccr}
\hline \hline Star & $V^{\mathrm{a}}$ & $B-V^{\mathrm{a}}$ & $\begin{array}{c}\mathrm{RV} \\
{\left[\mathrm{km} \mathrm{s}^{-1}\right]}\end{array}$ & $\begin{array}{c}\mathrm{EW} \\
{[\mathrm{mA}]}\end{array}$ & He I & $\begin{array}{c}\mathrm{T}_{\text {eff }} \\
{[\mathrm{K}]}\end{array}$ & $\begin{array}{c}\log \mathrm{g} \\
{\left[\mathrm{cm} \mathrm{s}^{-2}\right]}\end{array}$ & {$[\mathrm{Fe} / \mathrm{H}]$} & {$[\mathrm{Na} / \mathrm{Fe}]$} & {$[\mathrm{Al} / \mathrm{Fe}]$} \\
\hline LEID 31095 & 13.10 & 1.059 & +244.5 & 56.6 & yes & 4658 & 1.65 & -1.76 & 0.35 & 1.20 \\
LEID 31152 & 13.20 & 1.046 & +234.9 & 171 & yes & 4637 & 1.67 & -1.74 & 0.26 & 0.62 \\
LEID 35228 & 12.95 & 1.077 & +224.7 & $\leq 18$ & no & 4563 & 1.54 & -1.87 & 0.34 & 1.06 \\
LEID 38269 & 13.40 & 1.077 & +250.5 & $\leq 3.5$ & no & 4607 & 1.74 & -1.16 & 0.09 & 0.20 \\
LEID 41236 & 13.36 & 1.064 & +238.4 & 124 & yes & 4607 & 1.72 & -1.50 & 0.44 & 1.28 \\
LEID 43010 & 13.01 & 1.042 & +241.8 & $\leq 15.3$ & no & 4592 & 1.57 & -1.85 & -0.26 & $\leq 0.16$ \\
LEID 54018 & 13.48 & 1.042 & +246.1 & 196 & yes & 4565 & 1.74 & -1.50 & 0.59 & 1.11 \\
LEID 54064 & 13.27 & 1.048 & +233.6 & $\leq 9.2$ & no & 4741 & 1.76 & -1.86 & -0.14 & $\leq 0.36$ \\
LEID 54084 & 13.21 & 1.044 & +213.8 & 89.5 & yes & 4745 & 1.74 & -1.79 & 0.37 & 1.12 \\
LEID 55056 & 13.44 & 1.044 & +208.6 & $\leq 12$ & no & 4769 & 1.84 & -1.76 & 0.63 & 1.37 \\
LEID 58030 & 12.99 & 1.074 & +223.8 & $\leq 19.6$ & no & 4650 & 1.60 & -1.87 & -0.27 & $\leq 0.31$ \\
LEID 63027 & 13.02 & 1.058 & +237.1 & $\leq 13.9$ & no & 4675 & 1.63 & -1.79 & -0.09 & $\leq 0.24$ \\
\hline
\end{tabular}

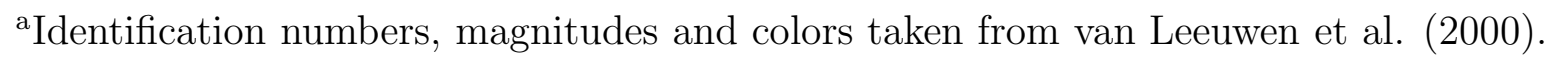


Table 2. Atomic Parameters and Equivalent Widths ${ }^{\mathrm{a}}$

\begin{tabular}{|c|c|c|c|c|c|c|c|c|c|c|c|c|c|c|}
\hline Ion & $\lambda[\AA]$ & $\log g f$ & 31095 & 31152 & 35228 & 38269 & 41236 & 43010 & 54018 & 54064 & 54084 & 55056 & 58030 & $63027^{\mathrm{b}}$ \\
\hline $\mathrm{Fe}$ II & 4620.52 & -3.23 & 38.3 & 30.1 & 36.3 & 51.8 & 42.2 & 35.5 & 41.1 & 32.7 & 35.7 & 39.2 & 31.9 & 36.4 \\
\hline $\mathrm{Fe}$ II & 4670.17 & -4.05 & 14.0 & 15.3 & 16.7 & 23.9 & 15.9 & 13.5 & 19.7 & 12.9 & 14.8 & 10.3 & 14.3 & 18.7 \\
\hline $\mathrm{Fe}$ II & 5100.66 & -4.20 & 9.1 & $\ldots$ & $\ldots$ & 25.0 & 18.0 & 7.5 & 12.9 & 9.7 & 8.5 & 8.9 & $\ldots$ & 9.5 \\
\hline Fe II & 5132.67 & -4.01 & 9.6 & 8.2 & 8.6 & 25.0 & 17.1 & 9.9 & 11.1 & 8.2 & 9.9 & 9.7 & $\ldots$ & 11.0 \\
\hline Fe II & 5197.58 & -2.24 & 71.7 & 65.0 & 72.9 & 83.1 & 74.3 & 72.3 & 72.9 & 65.6 & 70.6 & 74.3 & 66.3 & 69.8 \\
\hline $\mathrm{Fe}$ II & 5234.63 & -2.10 & 75.1 & 70.7 & 73.7 & 81.7 & 79.2 & 71.9 & 74.3 & 71.4 & 70.0 & 75.3 & 70.7 & 73.9 \\
\hline $\mathrm{Fe}$ II & 5264.79 & -3.02 & 28.1 & 22.6 & 25.3 & 39.0 & 28.9 & 24.2 & 32.4 & 23.7 & 24.9 & 29.3 & 24.5 & 21.6 \\
\hline $\mathrm{Fe}$ II & 5991.38 & -3.57 & 15.0 & 17.1 & 15.3 & 30.4 & 18.1 & 16.2 & 19.3 & 15.6 & 16.7 & 16.6 & 16.3 & 15.7 \\
\hline Fe II & 6084.10 & -3.81 & 10.9 & 11.7 & 3.9 & 18.9 & 14.1 & 7.3 & 15.7 & 7.5 & 9.5 & 13.6 & 8.4 & 9.1 \\
\hline $\mathrm{Fe}$ II & 6416.93 & -2.68 & 15.3 & 13.9 & 15.0 & 22.7 & 20.2 & 14.7 & 20.6 & 13.0 & 15.0 & 13.2 & 10.3 & 14.3 \\
\hline $\mathrm{Fe}$ II & 6432.68 & -3.57 & 26.9 & 24.0 & 23.8 & 35.7 & 25.3 & 24.5 & 24.0 & 22.6 & 27.0 & 25.4 & 24.0 & 25.5 \\
\hline $\mathrm{Fe}$ II & 6456.39 & -2.13 & 48.4 & 38.6 & 42.5 & 49.2 & 50.6 & 38.2 & 46.4 & 37.0 & 40.4 & 46.7 & 39.3 & 42.4 \\
\hline Fe II & 6516.08 & -3.28 & 40.6 & 34.3 & 38.3 & 51.7 & 41.3 & 36.7 & 39.8 & 34.3 & 39.3 & 38.6 & 33.7 & 36.0 \\
\hline$\overline{\mathrm{Al} \mathrm{I}}$ & 6696.03 & -1.45 & 41.1 & 12.1 & 29.9 & 16.1 & 61.7 & 5.9 & 46.7 & 6.8 & 30.8 & 43.8 & 7.7 & 4.9 \\
\hline $\mathrm{Al} \mathrm{I}$ & 6698.67 & -1.87 & 20.4 & 7.5 & 12.5 & 9.8 & 35.9 & $\ldots$ & 30.3 & 2.1 & 14.5 & 23.4 & 2.9 & 4.6 \\
\hline $\mathrm{Al} \mathrm{I}$ & 7835.32 & -0.74 & 30.2 & 11.7 & 19.4 & 19.7 & 48.5 & 2 & 39.6 & 4.2 & 19.6 & 37.1 & 1.9 & 6 \\
\hline $\mathrm{Al} \mathrm{I}$ & 7836.13 & -0.45 & 31.0 & 13.3 & 26.8 & 18.7 & 57.3 & $\ldots$ & 53.3 & 3.7 & 28.8 & 44.7 & $\ldots$ & 1.8 \\
\hline $\mathrm{Na} \mathrm{I}$ & 4668.57 & -1.41 & 8.0 & 7.0 & 7.5 & 6.1 & 19.6 & 3.1 & 18.1 & 3.7 & 4.6 & 8.6 & $\cdots$ & $\cdots$ \\
\hline $\mathrm{Na} \mathrm{I}$ & 5682.65 & -0.70 & 36.9 & 30.4 & 27.2 & 54.9 & 60.3 & 13.2 & 78.7 & 12.5 & 29.5 & 44.9 & 10.3 & 15.6 \\
\hline $\mathrm{Na} \mathrm{I}$ & 5688.21 & -0.37 & 57.5 & 43.2 & 50.9 & 74.1 & 75.5 & 17.0 & 93.3 & 15.8 & 45.7 & 68.4 & 16.3 & 22.8 \\
\hline $\mathrm{Na} \mathrm{I}$ & 6154.23 & -1.56 & 8.7 & $<10.7^{\mathrm{c}}$ & 6.8 & 17.9 & 14.4 & $<5.1$ & 19.0 & 2.2 & 4.9 & $<13.6$ & 3.5 & $<5$ \\
\hline $\mathrm{Na} \mathrm{I}$ & 6160.75 & -1.26 & 12.5 & 13.6 & 13.5 & 26.6 & 25.7 & 5.0 & 30.2 & 5.3 & 12.6 & 22.6 & 2 & $<4$ \\
\hline
\end{tabular}

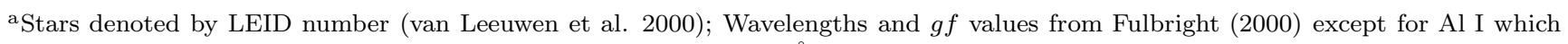
were taken from Jacobson et al. (2009); Equivalent widths given in $\mathrm{m} \AA$.

${ }^{\mathrm{b}} \mathrm{Na}$ abundance for LEID 63027 was also derived from the resonance lines ( $\lambda$ 5889.96 and $\left.\lambda 5895.94\right)$ and the abundance is in harmony with the values derived from the two Na lines listed below.

${ }^{\mathrm{c}}$ The $<$ symbol indicates the feature is blended, difficult to separate, and the value represents an upper limit to the equivalent width of the line. 